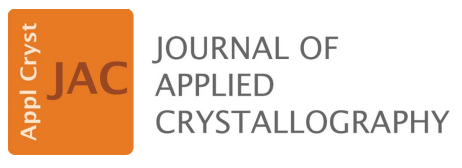

ISSN 1600-5767

\title{
System for in situ observation of three-dimensional structural changes in polymer films during uniaxial deformation. Corrigendum
}

\author{
Tsukasa Miyazaki, ${ }^{\text {a* }}$ Keisuke Shimokita, ${ }^{\text {b }}$ Hiroki Ogawa ${ }^{\mathrm{c}}$ and Katsuhiro Yamamoto ${ }^{\mathrm{d}}$
}

Received 19 January 2018

Accepted 29 January 2018

Keywords: small-angle $X$-ray scattering; wideangle X-ray diffraction; stress-strain relationships; three-dimensional structural evaluation.

\author{
${ }^{a}$ Neutron Science and Technology Center, Comprehensive Research Organization for Science and Society, 162-1 \\ Shirakata, Tokai, Naka, Ibaraki 319-1106, Japan, ${ }^{\mathbf{b}}$ Functional Base Products Sector, Nitto Denko Corporation, 18 \\ Hirayama, Nakahara, Toyohashi, Aichi 441-3194, Japan, ' Institute for Chemical Research, Kyoto University, Uji, Kyoto \\ 611-0011, Japan, and d Department of Materials Science, Nagoya Institute of Technology, Gokiso-cho, Showa-ku, \\ Nagoya 466-8555, Japan. *Correspondence e-mail: t_miyazaki@cross.or.jp
}

Ambiguities in the article by Miyazaki, Shimokita, Ogawa \& Yamamoto [ $J$. Appl. Cryst. (2015), 48, 1016-1022] are clarified.

In our previous paper (Miyazaki et al., 2015), we claimed that a complete three-dimensional structural evaluation can be performed by taking the edge and end views of a film during uniaxial deformation. However, this claim is not correct in the general situation, when a film is deformed with cylindrical symmetry during film stretching. Very little structural information can be obtained from the end view. On the assumption that, during film stretching, a system has a fiber symmetry which corresponds to the cylindrical symmetry around the stretching direction, detailed three-dimensional structural information such as the thickness of the lamellar crystallites and the fibril length and width has been obtained from the two-dimensional scattering pattern in the through view by projecting the scattering intensity onto the equatorial axis and the meridional one (Wu et al., 2001a,b).

However, if the complete analyses are to be applied to a film system during stretching, it must be guaranteed that the system has a fiber symmetry during stretching. In general, it is difficult to maintain a uniaxially deformed state during stretching using the usually available stretching apparatus. Especially in the case of polymeric film stretching with an extremely large strain, the system is likely to have in-plane orientation or double-axis orientation under the various stretching conditions with lower stretching temperatures, higher stretching speeds and higher ratios of the film width to the film length between the two sides of the clamped film. Therefore, for detailed analyses using the methods mentioned above, it is very important to elucidate the symmetry of the system in real time during deformation by observing the scattering pattern from the end view. The end view describes the symmetry around the stretching direction. If the film has a fiber symmetry during film stretching, an isotropic distribution of the scattering intensity around the stretching direction can be expected to evolve in the end-view two-dimensional scattering pattern with strain. 
In the previously reported in situ film stretching experiments, only two-dimensional scattering patterns in the through view have been obtained during film stretching by introducing the incident X-rays into the film from the direction normal to the film surface. It should be reported that our system is useful for actually confirming the fiber symmetry in the film during film stretching.

\section{References}

Miyazaki, T., Shimokita, K., Ogawa, H. \& Yamamoto, K. (2015). J. Appl. Cryst. 48, 1016-1022.

Wu, J., Schultz, J. M., Samon, J. M., Pangelinan, A. B. \& Chuah, H. H. (2001a). Polymer, 42, 7141-7151.

Wu, J., Schultz, J. M., Samon, J. M., Pangelinan, A. B. \& Chuah, H. H. (2001b). Polymer, 42, 7161-7170. 